\section{Acknowledgments}

We thank Lynne C. Weaver for her critical review of the literature and Ilda Moniz for her assistance.

Address correspondence to: Gregory A. Dekaban, BioTherapeutics Research Laboratory, Molecular Brain Research Group, Robarts Research Institute, Rm 2-12, 100 Perth Drive, London, Ontario, N6A 5K8 Canada. Phone: (519) 663-5777 ext. 24241; Fax: (519) 391-5789; E-mail: dekaban@robarts.ca.

1. Rossignol, S., Schwab, M., Schwartz, M., and Fehlings, M.G. 2007. Spinal cord injury: time to move? J. Neurosci. 27:11782-11792

2. Popovich, P.G., and Longbrake, E.E. 2008. Can the immune system be harnessed to repair the CNS? Nat. Rev. Neurosci. 9:481-493.

3. Riegger, T., et al. 2009. Immune depression syndrome following human spinal cord injury (SCI): a pilot study. Neuroscience. 158:1194-1199.

4. Rosas-Ballina, M., and Tracey, K.J. 2009. Cholinergic control of inflammation. J. Intern. Med. 265:663-679.
5. Lu, J., et al. 2009. Systemic inflammatory response following acute traumatic brain injury. Front. Biosci. 14:3795-3813

6. Ankeny, D.P., Guan, Z., and Popovich, P.G. 2009 $B$ cells produce pathogenic antibodies and impair recovery after spinal cord injury in mice. J. Clin. Invest. 119:2990-2999.

7. Dalakas, M.C. 2008. B cells as therapeutic targets in autoimmune neurological disorders. Nat. Clin. Pract. Neurol. 4:557-567.

8. Dalakas, M.C. 2008. Invited article: inhibition of B cell functions: implications for neurology. Neurology. 70:2252-2260

9. Corcione, A., et al. 2004. Recapitulation of B cell differentiation in the central nervous system of patients with multiple sclerosis. Proc. Natl. Acad. Sci.U. S. A. 101:11064-11069.

10. Waubant, E. 2008. Spotlight on anti-CD20. Int. MS J. 15:19-25.

11. Elkon, K., and Casali, P. 2008. Nature and functions of autoantibodies. Nat. Clin. Pract. Rheumatol. 4:491-498.

12. Matsushita, T, and Tedder, T.F. 2009. B-lymphocyte depletion for the treatment of multiple sclerosis: now things really get interesting. Expert Rev. Neurother. 9:309-312.

13. Lucin, K.M., Sanders, V.M., Jones, T.B., Malarkey, W.B., and Popovich, P.G. 2007. Impaired antibody synthesis after spinal cord injury is level dependent and is due to sympathetic nervous system dysregulation. Exp. Neurol. 207:75-84.

14. Kwidzinski, E., and Bechmann, I. 2007. IDO expression in the brain: a double-edged sword. J. Mol. Med. 85:1351-1359.

15. Oxenkrug, G.F. 2007. Genetic and hormonal regulation of tryptophan kynurenine metabolism: implications for vascular cognitive impairment, major depressive disorder, and aging. Ann. N. Y. Acad. Sci. 1122:35-49.

16. Kwidzinski, E., et al. 2005. Indolamine 2,3-dioxygenase is expressed in the CNS and down-regulates autoimmune inflammation. FASEB J. 19:1347-1349.

17. Xu, H., Zhang, G.X., Ciric, B., and Rostami, A. 2008. IDO: a double-edged sword for $\mathrm{T}(\mathrm{H}) 1 / \mathrm{T}(\mathrm{H}) 2$ regulation. Immunol. Lett. 121:1-6.

18. Mellor, A.L., and Munn, D.H. 2008. Creating immune privilege: active local suppression that benefits friends, but protects foes. Nat. Rev. Immunol. 8:74-80.

19. Scott, G.N., et al. 2009. The immunoregulatory enzyme IDO paradoxically drives B cell-mediated autoimmunity. J. Immunol. 182:7509-7517.

20. Gold, R., Stangel, M., and Dalakas, M.C. 2007. Drug insight: the use of intravenous immunoglobulin in neurology--therapeutic considerations and practical issues. Nat. Clin. Pract. Neurol. 3:36-44.

\title{
Reduced thymus activity and infection prematurely age the immune system
}

\author{
Ronald E. Gress ${ }^{1}$ and Steven G. Deeks ${ }^{2}$
}

1Experimental Transplantation and Immunology Branch, Center for Cancer Research, National Cancer Institute Bethesda, Maryland, USA. ${ }^{2}$ UCSF, San Francisco, California, USA.

The aging process affects all aspects of the immune system, particularly the $T$ cells. The immune system in older individuals is often characterized by lower $T$ cell numbers, lower naive/memory $T$ cell ratios, and lower $T$ cell diversity. Most measures of inflammation increase with age. Why this happens, and why there is so much person-to-person variability in these changes, is not known. In this issue of the JCI, Sauce and colleagues show that removal of the thymus during infancy results in premature onset of many of these age-associated changes to the immune system (see the related article beginning on page 3070 ). The effect of thymectomy was particularly notable in those individuals who acquired CMV infection. Data from this study, as well as data from other observational settings, suggest that reduced thymic function and persistent viral infections combine to accelerate a decline in immunologic function.

The aging process affects all organs, including the immune system. Immunologic aging, generally referred to as "immunosenescence," clearly affects $\mathrm{T}$ cell function, but changes also occur in B cells, antigenpresenting cells, NK cells, and perhaps even

Conflict of interest: The authors have declared that no conflict of interest exists.

Citation for this article: J. Clin. Invest. 119:2884-2887 (2009). doi:10.1172/JCI40855. stem cells. With respect to T cells, the major age-associated changes include a decline in the total number of cells, a shift from a naive to a memory/effector $\mathrm{T}$ cell population, and a decline in $\mathrm{CD}^{+} \mathrm{T}$ cell receptor repertoire diversity. Generalized inflammation also increases with age, perhaps due to a loss of immunoregulatory function. These age-associated alterations result in immune compromise with potential susceptibilities to infection, autoimmunity, and neoplasia. Nearly all of the typical ageassociated complications, including heart disease, cancer, infection, dementia, and frailty, have been epidemiologically linked (but not necessarily causally linked) to the process of immunosenescence.

\section{Effect of thymectomy and CMV on immunosenescence}

Although the pathogenesis of immunosenescence has been well studied in experimental models, very little mechanistic work has been performed in humans. In this issue of the JCI, Sauce and colleagues studied a group of young adults who as infants underwent complete removal of their thymus during the surgical correction of a congenital heart defect (1). Compared with age-matched controls, the thymectomized adults had a number of classic age-associated immunologic abnormalities, including lower total $\mathrm{T}$ cell counts and a preferential loss of naive $\mathrm{T}$ cells. These observations were not surprising, because the thymus is an estab- 

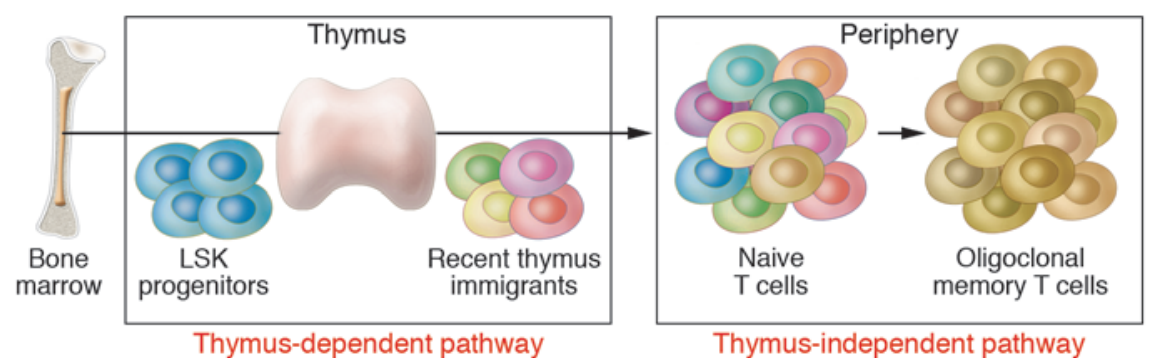

Figure 1

Mechanisms of naive T cell production. The naive T cell population can be maintained by ongoing $T$ cell production in the thymus. Hematopoietic stem cells in the bone marrow give rise to Lin-Sca- ${ }^{+{ }^{+} \mathrm{C}-\mathrm{Kit}^{+}}$(LSK) progenitors that settle in the thymus and give rise to naive $\mathrm{T}$ cells. With limited thymus function, proliferation of existing T cells in the periphery, which occurs via a thymus-independent pathway, can contribute to T cell numbers, but such T cell populations tend to be oligoclonal and have a memory phenotype. Complete surgical removal of the thymus early in life results in life-long reduction in naive T cell counts, as shown by Sauce and colleagues in their study in this issue of the $\mathrm{JCl}(1)$.

lished source of naive T cells (Figure 1) and a prior study of thymectomized persons found comparable results (2). What was novel was the observation that thymectomized adults exhibited a surprising degree of diversity in their immunologic profile, and a small subset ( 8 of $25,32 \%$ ) had a number of profound perturbations that are typically associated with very advanced aging (i.e., very low $\mathrm{CD}^{+} \mathrm{T}$ cell count, very low naive $\mathrm{T}$ cell frequency, reduced $\mathrm{CD}^{+}$ $\mathrm{T}$ cell repertoire, and high number of $\mathrm{CD} 57^{+}$immunosenescent memory T cells). These changes were comparable to that observed in a group of older individuals (more than 75 years old). CMV seropositivity among those who had undergone thymectomy was strongly (but not universally) associated with the presence of these profound age-associated immunologic abnormalities (Figure 2).

This association between CMV infection and immunologic aging (1) has been observed in other clinical scenarios. Among older individuals, the presence of CMV infection is associated with lower $\mathrm{CD}^{+} /$ $\mathrm{CD}^{+} \mathrm{T}$ cell ratios, lower naive/memory $\mathrm{T}$ cell ratios, reduced $\mathrm{T}$ cell proliferation, and an oligoclonal expansion of memory effector cells (3). Many of these changes have been associated with increased morbidity and mortality among older adults $(4,5)$. Among HIV-infected persons, CMV infection is predictive of progression to non-CMV end-organ disease and death (6). This effect may be related to the complex effect of CMV on the immune system in these already-immunocompromised patients (7). $\mathrm{CD}^{+} \mathrm{T}$ cell repertoire skewing in the period following an autologous hematopoietic stem cell transplant has also been reported to be associated with CMV infection (8). All of these apparently CMV-susceptible populations share one consistent characteristic: strong evidence for compromised thymic function and/or reduced ability to generate thymus-derived naive $\mathrm{CD}^{+}$and $\mathrm{CD}^{+} \mathrm{T}$ cells.

It is well known that CMV causes an extraordinary expansion of CMV-specific $\mathrm{CD}^{+} \mathrm{T}$ cells. This is even true in immunocompetent young adults (9). It is also now well established that thymectomy during childhood results in sustained immunologic abnormalities, such as lower $\mathrm{T}$ cell counts and a lower naive/memory $\mathrm{T}$ cell ratio. As now reported by Sauce et al. (1), this is apparently even true in CMV-uninfected adults who have undergone a thymectomy. The real question raised by the Sauce et al. study is what accounts for the apparent interaction between these two risk factors. In other words, are the effects of CMV and lack of thymic function more than additive and, if so, why? At this point in time, we can only speculate on these questions, but a number of diverse observations indicate sustained inflammation as a potential factor. Experimental ablation of the thymus in mice results in increased pathogen burden (including increased gut microbial translocation) and chronic inflammation (10). The surgical removal of the thymus at birth results in higher levels of inflammatory biomarkers, as reported by Sauce et al. (1), and the tically is associated with reduced $\mathrm{T}$ cell activation (11). The capacity of CMV to cause high-level, antigen-specific and perreversal of thymic dysfunction therapeu- haps non-antigen-specific inflammatory responses is well known $(9,12)$. Theoretically, the lack of a thymus and the presence of CMV may result in sustained inflammation and high-level $\mathrm{T}$ cell turnover, resulting eventually in an exhausted ability of an already compromised immune system to maintain a normal healthy adaptive immune system. Although the potential link between inflammation and immunologic "exhaustion" and/or immunosenescence is often discussed in the literature, no definitive proof for this concept exists, at least in humans $(13,14)$.

\section{Limitations in current literature}

The current study by Sauce and colleagues has a number of other limitations that deserve comment (1). First, given the crosssectional nature of the study, it is possible (albeit unlikely) that the presence of a thymectomy-associated immunosenescent phenotype increased the risk of acquiring $\mathrm{CMV}$, rather than CMV infection causing these immunologic changes. Second, the study did not specifically compare CMVseropositive thymectomized adults with age-matched, CMV-seropositive controls. A follow-up study focusing on these populations - with pre- and post-CMV infection samples analyzed, if possible - would provide more definitive evidence for the hypothesis that the lack of thymic function and the acquisition of CMV result in a synergistic effect on immune function. Third, although the sample size was reasonable, many of the novel findings are derived from the small subset of seven CMV-seropositive thymectomized adults. Larger studies are now warranted. Finally, as noted by the authors, no one has shown that thymectomy in humans results in a higher risk of significant morbidity and mortality. Indeed, $T$ cell function, as defined by antigen-specific cytokine production, was comparable in the age-matched thymectomized and control populations (1). Although it is reasonable to assume that the combination of a prior thymectomy and CMV infection will result in higher than expected rates of a number of age-associated conditions, this will need to be explicitly studied as the current generation of thymectomized adults reach older age.

\section{Clinical implications}

These data have a number of clinical implications (1). First, as stated by Sauce and colleagues, the surgical removal of thymus during pediatric heart surgery 

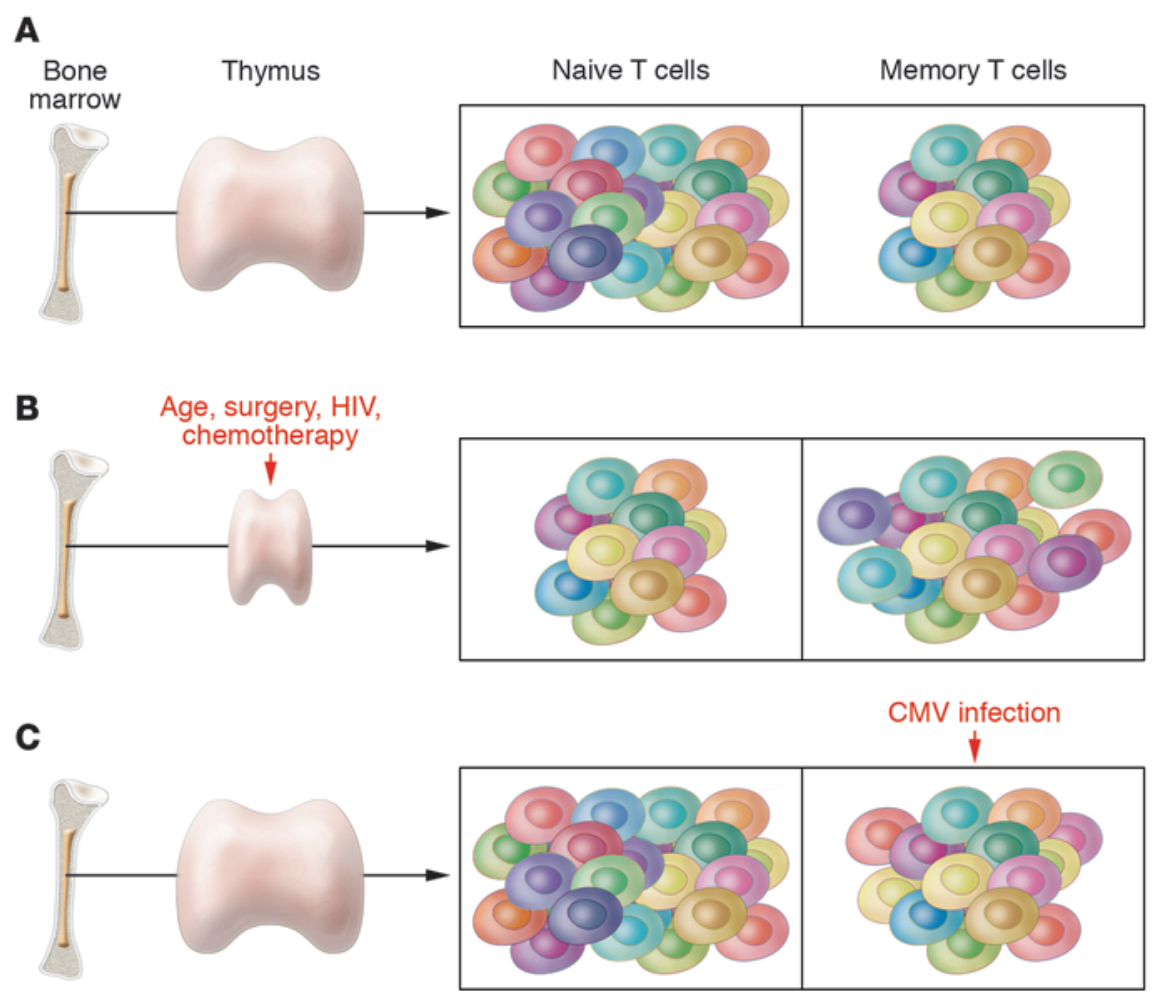

D

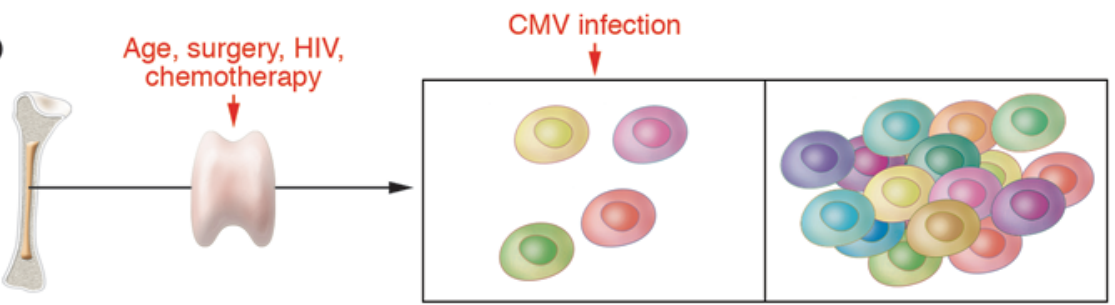

might be revisited, if technically feasible. Leaving any remnants of the thymus intact may prove over decades to be beneficial. Second, more urgency is needed for the development of a vaccine to prevent CMV infection and/or the development of new therapeutic approaches to treat or even eradicate CMV. To date, most vaccine studies have focused on women of childbearing age (to prevent congenital CMV infection) and most therapeutic trials have focused on those immunocompromised patients who are at risk for end-organ CMV disease (i.e., post-transplantation, advanced HIV disease). Given the emerging dataset implicating CMV as a key factor in driving immunologic aging, and given the growing data that these immunosenescent findings have clinical implications (at least in the very old), any intervention that can prevent or cure CMV infection might prove to have implications for a large segment of our population. Third, an antiinflamma- tory agent that could be given indefinitely might prove to have benefit in delaying the combined effects of CMV infection and lack of thymic function on the aging process. Given the proinflammatory nature of this clinical scenario, it would not be surprising if such patients prove to be at an increased risk for many age- and inflammation-associated diseases, such as cardiovascular disease.

Perhaps the most promising area of investigation pertains to immune-based therapeutics that can enhance thymic function in adults. The maintenance of normal $\mathrm{T}$ cell immunity depends upon the generation of new $T$ cells and the proliferation of those cells already released into peripheral compartments - that is, thymus-dependent and thymus-independent peripheral expansion pathways (15). Both pathways may be manipulated therapeutically. Growth hormone and its associated biologic partner IGF-1 enhance thymopoiesis by increasing

\section{Figure 2}

Effect of thymectomy and CMV on markers of immunosenescence. (A) In the presence of normal thymic function and in the absence of CMV infection, the immune system ages slowly. $T$ cell counts and $T$ cell function remain high, and there is no dramatic shift in the high naive/memory $T$ cell ratio over time. (B) In the presence of a direct insult to thymic function (e.g., surgical removal, HIV infection), some age-associated changes to the immune system can emerge early in life. A lower than expected frequency of naive $T$ cells is the most common observation, as shown by Sauce and colleagues in this issue of the $\mathrm{JCl}$ (1). (C) In the presence of retained thymic function, CMV infection (and perhaps other persistent infections) results in dramatic expansion of CMV-specific CD8 ${ }^{+}$and $\mathrm{CD}^{+}$ memory $T$ cells. Over several decades, CMV infection can result in many of the classic findings associated with immunosenescence. (D) In the absence of normal thymic function, the acquisition of CMV infection during early life results in the premature onset of immunosenescence, at least as defined by marked reductions in naive $T$ cell counts. By the time such individuals reach early adulthood, their T cell population appears to share many similarities with the $\mathrm{T}$ cell population of very old adults (> 75 years old). the thymus epithelial cell compartment (16). Growth hormone has already shown promise in enhancing the thymic function of HIV-1-infected adults in clinical trials (11). It is known that androgen signaling blockade enhances thymus function in part by upregulation of expression of CCL25 on medullary thymus epithelium (17). Interfering with the androgen signaling pathway increases thymus function in humans as well as mice (18). IL-7 supports the peripheral expansion of existing naive $\mathrm{T}$ cell populations. In an initial phase I study of recombinant human IL-7 (rhIL-7) administration in humans, rhIL-7 disproportionately increased the numbers of naive and central memory cells. $\mathrm{CD}^{+} \mathrm{T}$ cell repertoire diversity also increased. These effects were age-independent and persisted after therapy $(19,20)$.

Although the use of immune-based therapeutics to accelerate immune reconstitution has suffered a number of major 
setbacks recently (21), the overwhelming clinical need for these interventions is clear. A definition of affordable, safe, and effective therapeutic intervention that can enhance $T$ cell renewal is clearly a challenge worth pursuing.

Address correspondence to: Steven G. Deeks, University of California, Ward 84, Building 80, 995 Potero Avenue, San Francisco, California 94110, USA. Phone: (415) 476-4082 ext. 404; Fax: (415) 476-6953; E-mail: sdeeks@php.ucsf.edu.

1. Sauce, D., et al. 2009. Evidence of premature immune aging in patients thymectomized during early childhood. J. Clin. Invest. 119:3070-3078.

2. Prelog, M., et al. 2009. Thymectomy in early childhood: significant alterations of the CD4(+) CD45RA(+)CD62L $(+) \mathrm{T}$ cell compartment in later life. Clin. Immunol. 130:123-132.

3. Hadrup, S.R., et al. 2006. Longitudinal studies of clonally expanded CD8 T cells reveal a repertoire shrinkage predicting mortality and an increased number of dysfunctional cytomegalovirusspecific $T$ cells in the very elderly. J. Immunol. 176:2645-2653.

4. Wikby, A., et al. 2006. The immune risk phenotype is associated with IL-6 in the terminal decline stage: findings from the Swedish NONA immune longitudinal study of very late life functioning. Mech. Ageing Dev. 127:695-704.

5. Strindhall, J., et al. 2007. No Immune Risk Profile among individuals who reach 100 years of age: findings from the Swedish NONA immune longitudinal study. Exp. Gerontol. 42:753-761.

6. Deayton, J.R., et al. 2004. Importance of cytomegalovirus viraemia in risk of disease progression and death in HIV-infected patients receiving highly active antiretroviral therapy. Lancet. 363:2116-2121.

7. Hsue, P.Y., et al. 2006. Increased carotid intimamedia thickness in HIV patients is associated with increased cytomegalovirus-specific T-cell responses. AIDS. 20:2275-2283.

8. Peggs, K.S., et al. 2003. Reconstitution of T-cell repertoire after autologous stem cell transplantation: influence of CD34 selection and cytomegalovirus infection. Biol. Blood Marrow Transplant. 9:198-205.

9. Sylwester, A.W., et al. 2005. Broadly targeted human cytomegalovirus-specific CD4+ and CD8+ T cells dominate the memory compartments of exposed subjects. J. Exp. Med. 202:673-685.

10. Bourgeois, C., Hao, Z., Rajewsky, K., Potocnik, A.J., and Stockinger, B. 2008. Ablation of thymic export causes accelerated decay of naive CD4 T cells in the periphery because of activation by environmental antigen. Proc. Natl. Acad. Sci.U. S. A. 105:8691-8696.

11. Napolitano, L.A., et al. 2008. Growth hormone enhances thymic function in HIV-1-infected adults. J. Clin. Invest. 118:1085-1098.

12. Barton, E.S., et al. 2007. Herpesvirus latency confers symbiotic protection from bacterial infection. Nature. 447:326-329.

13. Nikolich-Zugich, J. 2008. Ageing and life-long maintenance of T-cell subsets in the face of latent persistent infections. Nat. Rev. Immunol. 8:512-522.

14. Pawelec, G., et al. 2004. Is immunosenescence infectious? Trends Immunol. 25:406-410.

15. Hakim, F.T., et al. 2005. Age-dependent incidence, time course, and consequences of thymic renewal in adults. J. Clin. Invest. 115:930-939.

16. Chu, Y.W., et al. 2008. Exogenous insulin-like growth factor 1 enhances thymopoiesis predominantly through thymic epithelial cell expansion. Blood. 112:2836-2846.

17. Williams, K.M., et al. 2008. CCL25 increases thymopoiesis after androgen withdrawal. Blood. 112:3255-3263

18. Sutherland, J.S., et al. 2005. Activation of thymic regeneration in mice and humans following androgen blockade. J. Immunol. 175:2741-2753.

19. Sportes, C., et al. 2008. Administration of rhIL-7 in humans increases in vivo TCR repertoire diversity by preferential expansion of naive $\mathrm{T}$ cell subsets. J. Exp. Med. 205:1701-1714.

20. Levy, Y., et al. 2009. Enhanced T cell recovery in HIV-1-infected adults through IL-7 treatment. J. Clin. Invest. 119:997-1007.

21. Kuritzkes, D.R. 2009. Interleukin-2: trials and tribulations. J. Infect. Dis. 200:164-165.

\title{
Pin1 regulates parathyroid hormone mRNA stability
}

\author{
Rajiv Kumar \\ Division of Nephrology and Hypertension, Departments of Medicine, Biochemistry, and Molecular Biology, \\ Mayo Clinic and Foundation, Rochester, Minnesota, USA.
}

\begin{abstract}
Secondary hyperparathyroidism often occurs in chronic kidney disease (CKD) and vitamin $\mathrm{D}$ deficiency, resulting in increased fractures and mortality. Understanding factors that stimulate parathyroid hormone (PTH) synthesis is important for devising methods to treat this condition. Previous work has demonstrated that murine $P$ th $\mathrm{mRNA}$ levels are regulated by proteins that bind AU-rich elements (AREs) within the $3^{\prime}$ UTR region of $P t h$ mRNA and influence Pth mRNA stability. In this issue of the JCI, Nechama et al. demonstrate that in murine secondary hyperparathyroidism associated with CKD or Ca deficiency, the activity of Pin1, a peptidyl-prolyl isomerase, is reduced (see the related article beginning on page 3102). Reduced Pin 1 activity resulted in the phosphorylation and degradation of an ARE-binding protein, K-homology splicing regulator protein (KSRP), which normally enhances the degradation of $P t h$ mRNA. The activity of other ARE-binding proteins, such as AU-rich binding factor 1 (AUF1), that increase Pth mRNA stability, was increased, thereby increasing PTH synthesis. This work suggests new ways by which to regulate PTH synthesis in secondary hyperparathyroidism.
\end{abstract}

Conflict of interest: The author has declared that no conflict of interest exists.

Citation for this article: J. Clin. Invest. 119:2887-2891 (2009). doi:10.1172/JCI40784.

\section{RNA processing in the cytoplasm regulates RNA concentrations}

Following transcription, nascent RNA is processed by $5^{\prime}$ methyl capping, splicing, cleavage, and polyadenylation in the nucleus (1-4) (Figure 1). RNA is exported from the nucleus and associates with various cellular structures prior to association with the ribosome. In the cytoplasm, RNA transcripts interact with RNA-binding proteins that influence RNA half-life and stability within the cell (5-8) (Figure 1). RNA-binding proteins (Table 1) associate with sequence-specific elements (adenine- and uridine-rich elements [AREs]) either within the coding or, more usually, within the $3^{\prime}$ UTRs of RNA. AREs regulate the rate at which mRNAs are degraded in cells and were first described as important elements involved in the regulation of the stability and half-life of protooncogene and cytokine mRNAs (1,9-12). AREs often contain overlapping adenine- and uridine-containing AUUUA pentamers that are found in U-rich regions within the $3^{\prime}$ UTRs of various genes (13). Three classes of AREs have been described: class I AREs contain 